\title{
Performance Bounds of Network Coding Aided Cooperative Multiuser Systems
}

\author{
Hung Viet Nguyen, Soon Xin Ng, and Lajos Hanzo, Fellow, IEEE
}

\begin{abstract}
In this contribution, we derive the upper and lower Frame Error Ratio (FER) performance bounds of cooperative multiuser communications systems using network coding. Our Monte Carlo simulation based results confirm the accuracy of our derivation.
\end{abstract}

Index Terms-Cooperative systems, multiuser systems, network coding.

\section{INTRODUCTION}

$\mathbf{N}$ ETWORK coding has been proved to be capable of increasing the achievable throughput, while minimizing both the amount of energy as well as delay of packets travelling through the network [1]-[3].

The Dynamic Network Code (DNC) concept proposed in [4]-[6] was extended in [7], [8] in order to introduce Generalized Dynamic Network Codes (GDNC). In GDNC aided systems, each user is allowed to broadcast several (as opposed to a single in [4]-[6]) information frames (IF) of its own during the broadcast phase (BP) via orthogonal channels, as well as to transmit several nonbinary linear combinations, which are also considered as parity frames (PFs), during the cooperative phase (CP) via orthogonal channels. The FER performance of the GDNC scheme was determined in [7], [8] by calculating the rank of the matrix characterising GDNCs. This method, which we refer to as the Purely Rank-Based Method (PRBM), always provides an optimistic estimate of the attainable FER performance of GDNCs.

Based on this background, the novel contribution of this letter is that we derive both the upper and lower bounds of the outage probability of GDNCs. We verify the accuracy of the bounds using Monte-Carlo simulations.

\section{SYSTEM MODEL}

Let us initially describe a simple system having $M=2$ users communicating with a BS [4], where a transmission session consists of 4 phases, namely the BPs $B_{1}$ and $B_{2}$, as well as the CPs $C_{1}$ and $C_{2}$. In the transmission session, each user transmits $k_{1}=1 \mathrm{IF}$ during the corresponding $\mathrm{BP}$ and $k_{2}=1 \mathrm{PF}$

Manuscript received March 23, 2011; accepted April 15, 2011. Date of publication April 25, 2011; date of current version June 09, 2011. This work was supported by the Vietnamese International Education Development (VIED) fund and the EU under the auspices of the OPTIMIX project and also by the RC-UK under the India-UK Advanced Technology Centre I. The associate editor coordinating the review of this manuscript and approving it for publication was Dr. Min Wu.

The authors are with the School of ECS, University of Southampton, SO17 1BJ, U.K. (e-mail: hvn08r@ecs.soton.ac.uk; sxn@ecs.soton.ac.uk; 1h@ecs.soton.ac.uk).

Digital Object Identifier 10.1109/LSP.2011.2146773 during the corresponding $\mathrm{CP}$ according to the transfer matrix $\boldsymbol{G}_{2 \times 4}[4]-[6]$

$$
\boldsymbol{G}_{2 \times 4}=\left[\begin{array}{cc|cc}
1 & 0 & 1 & 1 \\
0 & 1 & 1 & 2
\end{array}\right]
$$

where the PF transmitted by User 1 (or User 2) during the CP $C_{1}$ (or $C_{2}$ ) is given by $\mathrm{PF}=\boldsymbol{G}_{2 \times 4}(1,3) I_{1}(1)+\boldsymbol{G}_{2 \times 4}(2,3) I_{2}(2)$ (or PF $=\boldsymbol{G}_{2 \times 4}(1,4) I_{1}(1)+\boldsymbol{G}_{2 \times 4}(2,4) I_{2}(2)$ ). The variable $I_{i}(i), i=[1,2]$, represents the IF transmitted by User $i$ during the BP $B_{i}$. For simplicity, we refer to a single transmission phase as a time slot (TS), in which a user transmits a single frame.

To elaborate further, let us define $\boldsymbol{G}_{2 \times 4}^{\prime}$ as the corresponding modified transfer matrix, where the terminology modified implies that the entries of $\boldsymbol{G}_{2 \times 4}^{\prime}$ are modified with respect to those of the original transfer matrix $\boldsymbol{G}_{2 \times 4}$ of (1) according to the success/failure of each transmission phase within an actual transmission session. If all the frames transmitted within the session are successfully decoded, the transmission session can be equivalently represented by the modified transfer matrix $\boldsymbol{G}_{2 \times 4}^{\prime}=\boldsymbol{G}_{2 \times 4}$, where $\boldsymbol{G}_{2 \times 4}^{\prime}(i, i)=\boldsymbol{G}_{2 \times 4}(i, i), i=[1,2]$ represents the successful decoding of the IF $I_{i}(i)$ at the BS. According to [7], [8], having $\boldsymbol{G}_{2 \times 4}^{\prime}(1,3)=\boldsymbol{G}_{2 \times 4}(1,3)$ (or $\boldsymbol{G}_{2 \times 4}^{\prime}(2,4)=\boldsymbol{G}_{2 \times 4}(2,4)$ ) means that the PF transmitted by User 1 (or User 2) was successfully decoded at the BS. Moreover, having $\boldsymbol{G}_{2 \times 4}^{\prime}(2,3)=\boldsymbol{G}_{2 \times 4}(2,3)$ (or $\boldsymbol{G}_{2 \times 4}^{\prime}(1,4)=\boldsymbol{G}_{2 \times 4}(1,4)$ ) indicates that the IF $I_{2}(2)$ (or $\left.I_{1}(1)\right)$ was successfully decoded by User 1 (or User 2), and that the PF transmitted by User 1 (or User 2) was successfully decoded at the BS.

Let us consider the following example of the actual transmission session, where ' $\rightarrow$ ' represents the transmission direction, while ' $=1$ ' (or ' $=0$ ') above the arrows means that the frame was successfully (or unsuccessfully) recovered at the destination:

$$
\begin{aligned}
B_{1}: \boldsymbol{G}_{2 \times 4}^{\prime}(1,3)=\boldsymbol{G}_{2 \times 4}(1,3), & {[\text { User } 1 \stackrel{=0}{\longrightarrow} B S]: \boldsymbol{G}^{\prime}(1,1)=0, } \\
& {[\text { User } 1 \stackrel{=1}{\longrightarrow} \text { User } 2]: \boldsymbol{G}_{2 \times 4}^{\prime}(1,4)=\boldsymbol{G}_{2 \times 4}(1,4) ; } \\
B_{2}: \boldsymbol{G}_{2 \times 4}^{\prime}(2,4)=\boldsymbol{G}_{2 \times 4}(2,4), & {[\text { User } 2 \stackrel{=0}{\longrightarrow} B S]: \boldsymbol{G}^{\prime}(2,2)=0, } \\
& {\left[\text { User } 2 \stackrel{=1}{\longrightarrow} \text { User 1]: } \boldsymbol{G}_{2 \times 4}^{\prime}(2,3)=\boldsymbol{G}_{2 \times 4}(2,3) ;\right.} \\
C_{1}: & {[\text { User } 1 \stackrel{=0}{\longrightarrow} B S]: \boldsymbol{G}_{2 \times 4}^{\prime}(i, 3)=0, i=1,2 ; } \\
C_{2}: & {[\text { User } 2 \stackrel{=1}{\longrightarrow} B S]: \boldsymbol{G}_{2 \times 4}^{\prime}(i, 4) \text { unchanged, } i=1,2 . }
\end{aligned}
$$

This example results in the $\boldsymbol{G}_{2 \times 4}^{\prime}$ given by

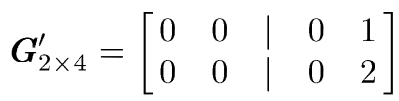




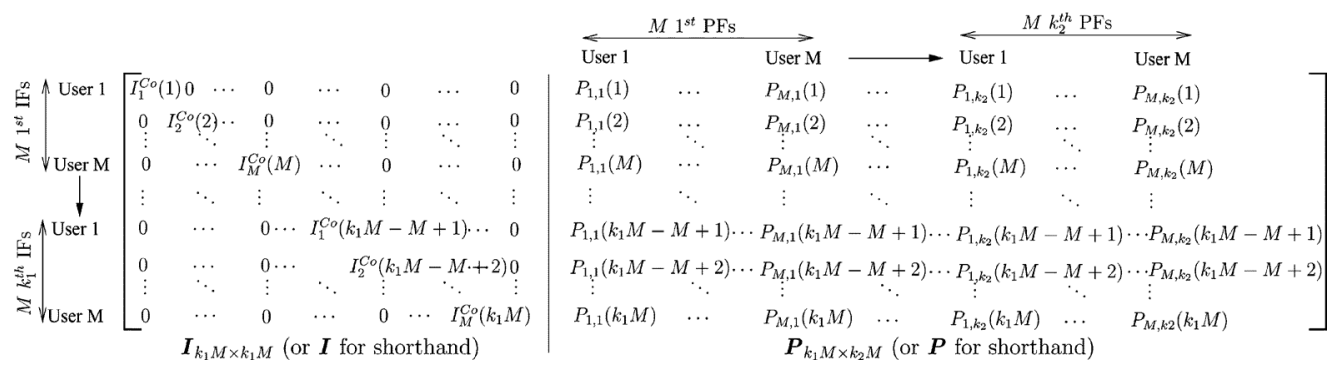

Fig. 1. Transfer matrix $\boldsymbol{G}_{k_{1} M \times k_{1} M+k_{2} M}$ illustrating a transmission session of the system having $M$ users transmitting in $\left(k_{1} M+k_{2} M\right)$ phases.

where the diagonal elements " 1 " at the left of (1) become "0" owing to the unsuccessful $[$ User $1 \stackrel{=0}{\longrightarrow} B S]$ and $[$ User $2 \stackrel{=0}{\longrightarrow} B S]$ transmissions during the BPs $B_{1}$ and $B_{2}$, respectively. The " 0 " elements in the third column of (3) indicate the unsuccessful [User $1 \stackrel{=0}{\longrightarrow} B S]$ transmission during the $\mathrm{CP} C_{1}$.

Let us now generalise this model. The transfer matrix $\boldsymbol{G}_{k_{1} M \times k_{1} M+k_{2} M}$ (or $\boldsymbol{G}$ for shorthand) in Fig. 1 comprising the identity matrix $\boldsymbol{I}_{k_{1} M \times k_{1} M}$ (or $\boldsymbol{I}$ for shorthand) and the parity matrix $\boldsymbol{P}_{k_{1} M \times k_{2} M}$ (or $\boldsymbol{P}$ for shorthand) represents a transmission session of the system, where all the frames transmitted during that session are successfully decoded. Accordingly, the binary flag $I_{m}^{C o}(t)$ seen in Fig. 1 represents the success or failure of the IF decoding at the BS, namely the IF $I_{m}(t)$, $t=\left[m, M+m, \ldots,\left(k_{1}-1\right) M+m\right]$, transmitted by User $m$, $m \in\{1, \ldots, M\} . I_{m}^{C o}(t)$ is set according to

$$
I_{m}^{C o}(t)= \begin{cases}1, & \text { if } I_{m}(t) \text { is successfully recovered } \\ 0, & \text { otherwise. }\end{cases}
$$

The $k_{2}$ PFs transmitted by each of the $M$ users contain nonbinary linear combinations of its own IFs with the successfully decoded IFs from the set of $k_{1}(M-1)$ IFs transmitted by the $(M-1)$ other users. The variable $P_{m, s}(t)$ in Fig. 1 corresponds to the parity coefficient of the IF $I_{r}(t)$ contained in the $s$ th PF transmitted by User $m$ during the CP $[M(s-1)+m]$, $s \in\left\{1, \ldots, k_{2}\right\}$. The index $r$ is determined by the rule that we get $r=M$ if we have $t \bmod M=0$, otherwise we get $r=t \bmod M$. Let us denote the corresponding entry of $P_{m, s}(t)$ in the modified matrix $\boldsymbol{G}^{\prime}$ as $P_{m . s}^{\prime}(t)$, which is determined by

$$
P_{m, s}^{\prime}(t)=\left\{P_{m, s}(t) \quad: r=m .\right.
$$

Then, for the case that we have $r \neq m$, the entry $P_{m, s}^{\prime}(t)$ is specified by

$$
P_{m, s}^{\prime}(t)=\left\{\begin{array}{ll}
P_{m, s}(t) & : \text { User } r \stackrel{=1}{\longrightarrow} \text { User } m \\
0 & : \text { User } r \stackrel{=0}{\longrightarrow} \text { User } m
\end{array} .\right.
$$

The column $[M(s-1)+m]$ of the parity matrix $\boldsymbol{P}$ shown in Fig. 1 contains the set of parity coefficients valid for the $s$ th PF transmitted by User $m$ during the CP $[M(s-1)+m]$. Hence, the entire column $P_{m, s}^{\prime}(t), \forall t=\left[1,2, \ldots, k_{1} M\right]$ will be set to zeros, if the BS could not sucessfully receive the $s$ th PF:

$$
P_{m, s}^{\prime}(t)=0, \forall t=\left[1,2, \ldots, k_{1} M\right]: \text { User } m \stackrel{s^{t h} P F=0}{\longrightarrow} \text { BS. }
$$

\section{ReCOVERY OF IFs AT THE BS Versus the PRBM}

As the system proceeds through an actual transmission session, the corresponding modified transfer matrix $\boldsymbol{G}^{\prime}$ consisting of its identity matrix $\boldsymbol{I}^{\prime}$ and its parity matrix $\boldsymbol{P}^{\prime}$ is formed, where $\boldsymbol{I}^{\prime}$ is generated from (4), while $\boldsymbol{P}^{\prime}$ is determined in turn by (5), (6) and (7). The frames successfully received at the BS can be represented as

$$
\text { (a) } \boldsymbol{X} \boldsymbol{I}^{\prime}=\boldsymbol{Y}_{I^{\prime}}, \text { (b) } \boldsymbol{X} \boldsymbol{P}^{\prime}=\boldsymbol{Y}_{P^{\prime}}
$$

where $\boldsymbol{X}=\left\{I_{1}(1), I_{2}(2), \ldots, I_{M}\left(k_{1} M\right)\right\}$ is a matrix representing the IFs transmitted by the $M$ users during the transmission session of the system, while the matrices of $\boldsymbol{Y}_{I^{\prime}}$ and $\boldsymbol{Y}_{P^{\prime}}$ represent the frames successfully received at the BS during the BPs and CPs, respectively. In line with [7], [8], we assume that the BS is aware of how each PF was constructed, hence $\boldsymbol{G}^{\prime}$ is known at the BS. Since the matrix $\boldsymbol{I}^{\prime}$ may be different from $\boldsymbol{I}$, the BS can certainly recover a set $\boldsymbol{X}_{I^{\prime}}$ of IFs, which is a subset of $\boldsymbol{X}$, from $\boldsymbol{Y}_{I^{\prime}}$ as

$$
\boldsymbol{X}_{I^{\prime}}=\boldsymbol{Y}_{I^{\prime}}
$$

Substituting $\boldsymbol{X}_{I^{\prime}}$ given by (9) into (8b) we have

$$
\left(\boldsymbol{X}-\boldsymbol{X}_{I^{\prime}}\right) \boldsymbol{P}^{\prime}=\boldsymbol{Y}_{P^{\prime}}-\boldsymbol{X}_{I^{\prime}} \boldsymbol{P}^{\prime} .
$$

Then, a set $\tilde{\boldsymbol{X}}_{P^{\prime}}$ of IFs is retrieved from (10) by using the Gaussian elimination algorithm. Ultimately, the entire set of IFs recovered at the BS is $\tilde{\boldsymbol{X}}_{P^{\prime}} \cup \boldsymbol{X}_{I^{\prime}}$ out of the $\boldsymbol{X}$ of IFs.

Let us characterise the system's optimistic performance estimated by the PRBM employed in [7], [8] by recalling the example detailed in (1), (2) and (3). According to the prediction of the PRBM, the BS can recover $\operatorname{Rank}\left(\boldsymbol{G}_{2 \times 4}^{\prime}\right)=1 \mathrm{IF}$, where $\boldsymbol{G}_{2 \times 4}^{\prime}$ is given in (3). However, in fact the BS cannot recover any IF, because we cannot determine unambiguously two IFs, i.e., both $I_{1}(1)$ and $I_{2}(2)$, from a single equation, which is inferred from (9) and (10) as $I_{1}(1)+2 I_{2}(2)$.

\section{Outage Probability}

Let $U_{m, t}$ be a set of user indices corresponding to the specific users that succeeded in correctly recovering an IF $I_{m}(t)$ transmitted by User $m$ during TS $t$. Let us denote the number of members in the user set $U_{m, t}$ by $\left\|U_{m, t}\right\|$. Furthermore, let the complement set of $U_{m, t}$ be $U_{m, t}^{*}$. We always have $\left\|U_{m, t}^{*}\right\|=$ $M-\left\|U_{m, t}\right\|, 1 \leq\left\|U_{m, t}\right\| \leq M$. Then, according to [4]-[8], there exist at least $\left(k_{1}\left\|U_{m, t}\right\|+k_{2}\left\|U_{m, t}\right\|\right)$ frames, which contain the IFs transmitted by all the users in the set $U_{m, t}$. Accordingly, an outage is declared for the IF $I_{m}(t)$, when the direct transmission $I_{m}(t)$ and at least $\left\|U_{m, t}\right\| k_{2}$ out of the remaining 
$\left(k_{1}\left\|U_{m, t}\right\|-1+\left\|U_{m, t}\right\| k_{2}\right)$ received frames are in outage. This occurs with a probability of [7], [8]

$$
P_{o, m}\left(U_{m, t}^{*}\right)=\sum_{i=0}^{Q} \underbrace{\left(\begin{array}{c}
Q+K \\
K+i
\end{array}\right) \frac{P_{e}^{K+i+1}}{\left(1-P_{e}\right)^{i-Q}}}_{=T_{o, m}(i)}
$$

where $\left(\begin{array}{l}n \\ k\end{array}\right)$ is the binomial coefficient, while we have $Q=k_{1}\left\|U_{m, t}\right\|-1$ and $K=k_{2}\left\|U_{m, t}\right\|$, provided that $P_{e}$ is the outage probability of the single link as defined in [9].

Note that there might be more than $\left(k_{1}\left\|U_{m, t}\right\|+k_{2}\left\|U_{m, t}\right\|\right)$ frames [4]-[8], which contain the IFs transmitted by all users of the set $U_{m, t}$. If the availability of those extra frames is taken into account, we will have the actual outage probability $P_{o, m}^{A c}\left(U_{m, t}^{*}\right)$ for the IF $I_{m}(t)$, which always satisfies

$$
P_{o, m}^{A c}\left(U_{m, t}^{*}\right) \leq P_{o, m}\left(U_{m, t}^{*}\right) .
$$

Notably, $P_{o, m}^{A c}\left(U_{m, t}^{*}\right)$ is the outage probability for a given $U_{m, t}^{*}$. The system's total outage probability $P_{o}$ for all possible sets of $U_{m, t}^{*}$ can be calculated by

$$
P_{o}=\sum_{\left\|U_{m, t}^{*}\right\|=0}^{M-1} \frac{P_{e}^{\left\|U_{m, t}^{*}\right\|}}{\left(1-P_{e}\right)^{\left\|U_{m, t}^{*}\right\|-M+1}} P_{o, m}^{A c}\left(U_{m, t}^{*}\right) .
$$

\section{A. The Upper Bound}

The ratio of two successive terms in (11), namely that of $T_{o, m}(j)$ and $T_{o, m}(j+1)$, may be computed as

$$
R_{o, m}(j)=\frac{T_{o, m}(j+1)}{T_{o, m}(j)}=\frac{(Q-j) P_{e}}{(K+j+1)\left(1-P_{e}\right)}
$$

where $j \in\{0, \ldots, Q-1\}$. Then, we can infer that

$$
R_{o, m}(Q-1) \leq R_{o, m}(j) \leq R_{o, m}(0) .
$$

By exploiting a series expansion, we can rewrite (11) as

$$
P_{o, m}\left(U_{m, t}^{*}\right)=T_{o, m}(0) \frac{1-\left[R_{o, m}(j)\right]^{Q}}{1-R_{o, m}(j)} .
$$

By exploiting (15) in (16) and substituting $T_{o, m}(0), R_{o, m}(0)$ and $R_{o, m}(Q-1)$ obtained from (11) and (14), we arrive at

$$
\begin{gathered}
P_{o, m}\left(U_{m, t}^{*}\right) \leq \frac{\left(\begin{array}{c}
Q+K \\
K
\end{array}\right)}{P_{e}^{-(K+1)}} \underbrace{\frac{\left(\beta P_{e}\right)^{Q+1}-\left(1-P_{e}\right)^{Q+1}}{\left(\beta P_{e}+P_{e}-1\right)}}_{=M_{u}}, \\
P_{o, m}\left(U_{m, t}^{*}\right) \geq \frac{\left(\begin{array}{c}
Q+K \\
K
\end{array}\right)}{P_{e}^{-(K+1)}} \underbrace{\frac{\left(\alpha P_{e}\right)^{Q+1}-\left(1-P_{e}\right)^{Q+1}}{\left(\alpha P_{e}+P_{e}-1\right)}}_{=M_{l}}
\end{gathered}
$$

where we have $\beta=Q /(K+1)$ and $\alpha=1 /(K+Q)$. Let us now consider the scenario of $P_{e} \ll 1$. The term $M_{u}$ of (17) may be simplified to

$$
M_{u}<\underbrace{\frac{1-P_{e}}{1-P_{e}-\frac{E}{F+1} P_{e}}}_{=M_{u}^{\max }}
$$

where we have $E=\left(M k_{1}-1\right)$ and $F=M k_{2}$. Then, the inequality (17) becomes

$$
P_{o, m}\left(U_{m, t}^{*}\right)<\underbrace{\left(\begin{array}{c}
Q+K \\
K
\end{array}\right) P_{e}^{K+1} M_{u}^{\max }}_{=P_{o, m}^{\max }\left(U_{m, t}^{*}\right)} .
$$

By combining (12), (13) and (20), we can infer that

$$
P_{o}<\sum_{\left\|U_{m, t}^{*}\right\|=0}^{M-1}\left(\begin{array}{c}
Q+K \\
K
\end{array}\right) \underbrace{\frac{P_{e}^{K+1+\left\|U_{m, t}^{*}\right\|}}{\left(1-P_{e}\right)\left\|U_{m, t}^{*}\right\|-M+1} M_{u}^{\max }}_{=T_{o}^{U p p e r}\left(\left\|U_{m, t}^{*}\right\|\right)} .
$$

Let us then exploit the fact that $\left(\begin{array}{c}Q+K \\ K\end{array}\right) \leq\left(\begin{array}{c}E+F \\ F\end{array}\right)$ and pay more attention to the case that we have $\left\|U_{m, t}^{*}\right\|=M-1$, in order to further approximate (21) as

$$
\begin{gathered}
P_{o}<\underbrace{\left.\frac{\left(\begin{array}{c}
Q+K \\
K
\end{array}\right)-\left(\begin{array}{c}
E+F \\
F
\end{array}\right)}{\left[T_{o}^{U p p e r}\left(\left\|U_{m, t}^{*}\right\|\right)\right]^{-1}}\right|_{\left\|U_{m, t}^{*}\right\|=M-1}}_{=\Omega} \\
+\sum_{\left\|U_{m, t}^{*}\right\|=0}^{M-1}\left(\begin{array}{c}
E+F \\
F
\end{array}\right) T_{o}^{U p p e r}\left(\left\|U_{m, t}^{*}\right\|\right) .
\end{gathered}
$$

Let us consider the ratio $R_{o}$ of $T_{o}^{U p p e r}(i)$ and $T_{o}^{U p p e r}(i+1)$ in (22), which allows us to arrive at the following result:

$$
R_{o}=\frac{T_{o}^{U p p e r}(i)}{T_{o}^{U p p e r}(i+1)}=\frac{1-P_{e}}{P_{e}^{1-k_{2}}}, i \in\{0, \ldots, M-2\} .
$$

Similar to the manner of formulating $P_{o, m}\left(U_{m, t}^{*}\right)$ in (16) from (11) and (14), the inequality of (22) can be expressed as

$$
P_{o}<\underbrace{\Omega+\left(\begin{array}{c}
E+F \\
F
\end{array}\right) P_{e}^{M+k_{2}} M_{u}^{\max } \frac{1-R_{o}^{M}}{1-R_{o}}}_{=P_{o}^{\text {Upper }}}
$$

where we define $P_{o}^{U p p e r}$ as the strict upper bound of the system's outage probability $P_{o}$.

\section{B. The Lower Bound}

It may be inferred from (13) that

$$
P_{o}>\left.\frac{P_{e}^{\left\|U_{m, t}^{*}\right\|}}{\left(1-P_{e}\right)^{\left\|U_{m, t}^{*}\right\|-M+1}} P_{o, m}^{A c}\left(U_{m, t}^{*}\right)\right|_{\left\|U_{m, t}^{*}\right\|=0} .
$$

Note that we always have $\left.P_{o, m}^{A c}\left(U_{m, t}^{*}\right)\right|_{\left|\left\|U_{m, t}^{*}\right\|\right|=0}=$ $\left.P_{o, m}\left(U_{m, t}^{*}\right)\right|_{\left\|U_{m, t}^{*}\right\|=0}$, which can be extracted from (11) as

$$
\left.P_{o, m}^{A c}\left(U_{m, t}^{*}\right)\right|_{\left\|U_{m, t}^{*}\right\|=0}=\sum_{i=0}^{E}\left(\begin{array}{c}
E+F \\
F+i
\end{array}\right) \frac{P_{e}^{F+i+1}}{\left(1-P_{e}\right)^{i-E}} .
$$

By considering $E$ and $F$ in (26) as the special case of $Q$ and $K$ in (18), respectively, we can use (18) to obtain

$$
\left.P_{o, m}^{A c}\left(U_{m, t}^{*}\right)\right|_{\left\|U_{m, t}^{*}\right\|=0} \geq\left(\begin{array}{c}
E+F \\
F
\end{array}\right) P_{e}^{F+1} M_{l}^{\min }
$$


where we have $M_{l}^{\text {min }}=\left.M_{l}\right|_{Q=E, K=F}$. Upon substituting (27) into (25), we have

$$
P_{o}>\underbrace{\left(\begin{array}{c}
E+F \\
F
\end{array}\right) P_{e}^{F+1} \frac{M_{l}^{\mathrm{min}}}{\left(1-P_{e}\right)^{1-M}}}_{=P_{o}^{\text {Lower }}}
$$

where we define $P_{o}^{\text {Lower }}$ as the strict lower bound of $P_{o}$.

\section{System PARAMETERS AND DIVERSITY ORDER}

We assume that all the links in the system are supported by channels having the same $R=0.5$ to make our results comparable to those presented in [7], [8]. For notational convenience, we characterise the system by using the set of parameters $(R$, $\left.M, k_{1}, k_{2}, \boldsymbol{G}, R_{\text {info }}, D\right)$, where the system's overall information rate $R_{\text {info }}$ is expressed as [7], [8]

$$
R_{\text {info }}=\frac{k_{1}}{k_{1}+k_{2}}
$$

while the diversity order $D$ of the system is bounded [4]-[8]:

$$
M+k_{2} \leq D \leq M k_{2}+1 .
$$

The authors of [4]-[8] inferred the diversity order $D$ in (30) based on the following formula:

$$
D=\lim _{S N R \rightarrow \infty} \frac{-\log _{2} P_{o}}{\log _{2} S N R}
$$

where $S N R$ is the signal to noise power ratio, while the order of $P_{e}$ for the best and worst case $P_{o}$ value was estimated and used instead of $P_{o}$ itself, assuming the approximate formula [9] of $P_{e}=1-e^{\left(1-2^{R}\right) / S N R} \approx\left(2^{R}-1\right) / S N R$.

Similarly, we may infer from the $P_{o}^{U p p e r}$ formula of (24) that the most influential term is $P_{e}^{M+k 2}$. Likewise, it can be seen in the $P_{o}^{\text {Lower }}$ formula of (28) that the term having the most significant influence is $P_{e}^{M k_{2}+1}$. Hence, it may be seen that the upper and lower bounds of the probability $P_{o}$ are in harmony with the estimated diversity order given by (30).

\section{NumericAl Results AND Discussions}

In this section, we compare the upper and lower bounds of the system's outage probability $P_{o}$ with our numerical results in Fig. 2 . The results associated with $\boldsymbol{G}_{2 \times 4}$-based and $\boldsymbol{G}_{4 \times 8}$-based systems in [7], [8] are also presented for direct comparison with our results.

Accordingly, the $\boldsymbol{G}_{2 \times 4}$-based system is represented by ( $R=$ $0.5, M=2, k_{1}=1, k_{2}=1, \boldsymbol{G}_{2 \times 4}, R_{\text {info }}=0.5,3 \leq D_{2 \times 4} \leq$ 3 ), and the $\boldsymbol{G}_{4 \times 8}$-based one is characterized by ( $R=0.5, M=$ $\left.2, k_{1}=2, k_{2}=2, \boldsymbol{G}_{4 \times 8}, R_{\text {info }}=0.5,4 \leq D_{4 \times 8} \leq 5\right)$. The two systems are comparable, since they have the same $R, M$ and $R_{\text {info }}$ values. However, the more complex transfer matrix $\boldsymbol{G}_{4 \times 8}$ [7], [8] has a higher diversity order of $4 \leq D_{4 \times 8} \leq 5$ (as opposed to $3 \leq D_{2 \times 4} \leq 3$ ), hence it is associated with a higher

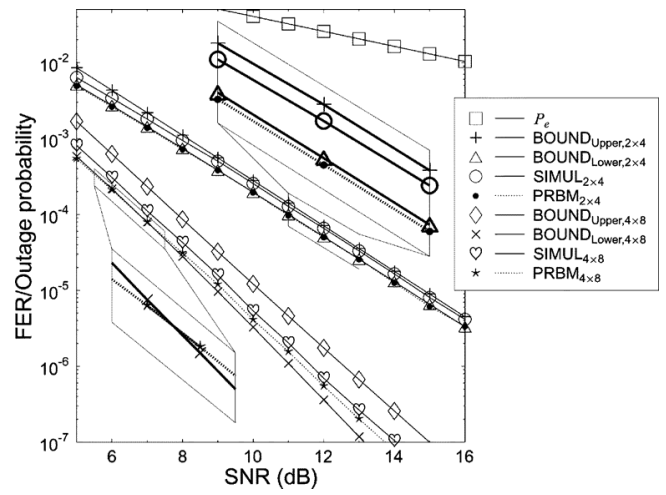

Fig. 2. Outage probability bounds and FER performance of the systems.

detection complexity at the BS. The differences in the diversity order are also reflected by the different slope of the bounds and the performance curves, as seen in Fig. 2.

Observe furthermore in Fig. 2 that the system's actual FER performance curve is always between the upper bound and lower bound. The PRBM always suggests a superior performance in comparison to actual one obtained by simulations, as demonstrated by the specific example of Section III. The PRBM-based performance estimate in fact violates in place the strict lower bounds.

In conclusion, the FER-performance upper and lower bounds of cooperative multiuser communications systems were derived. The system's FER-performance was also evaluated by Monte Carlo based simulations, in order to visualise the accuracy of those bounds.

\section{REFERENCES}

[1] P. Chou and Y. Wu, "Network coding for the internet and wireless networks," IEEE Signal Process. Mag., vol. 24, no. 5, pp. 77-85, 2007.

[2] R. Zhang and L. Hanzo, "Coding schemes for energy efficient multisource cooperation aided uplink transmission," IEEE Signal Process. Lett., vol. 16, pp. 438-441, May 2009.

[3] A. Ramasubramonian and J. Woods, "Multiple description coding and practical network coding for video multicast," IEEE Signal Process. Lett., vol. 17, no. 3, pp. 265-268, 2010.

[4] M. Xiao and M. Skoglund, "M-user cooperative wireless communications based on nonbinary network codes," in IEEE Information Theory Workshop on Networking and Information Theory, 2009. (ITW 2009), Jun. 2009, pp. 316-320.

[5] M. Xiao and M. Skoglund, "Design of network codes for multipleuser multiple-relay wireless networks," in IEEE Int. Symp. Information Theory, 2009. (ISIT 2009), Jun. 2009, pp. 2562-2566.

[6] M. Xiao and M. Skoglund, "Multiple-user cooperative communications based on linear network coding," IEEE Trans. Commun., Dec. 2010.

[7] J. L. Rebelatto, B. F. Uchôa-Filho, Y. Li, and B. Vucetic, "Generalized distributed network coding based on nonbinary linear block codes for multi-user cooperative communications," in 2010 IEEE Int. Symp. Information Theory (ISIT 2010), Jun. 2010, pp. 943-947.

[8] J. L. Rebelatto, B. F. Uchôa-Filho, Y. Li, and B. Vucetic, "Multi-user cooperative diversity through network coding based on classical coding theory," IEEE Trans. Inf. Theory [Online]. Available: http://arxiv.org/ abs/1004.2757, submitted for publication

[9] J. Laneman, D. Tse, and G. Wornell, "Cooperative diversity in wireless networks: Efficient protocols and outage behavior," IEEE Trans. Inf. Theory, vol. 50, pp. 3062-3080, Dec. 2004. 